\title{
Classifications of Cardiomyopathies - Frequent Updating, Modifications and Re-Classifications in the Era Of Molecular Medicine and the Past Decades MOHAMMAD ABU KAUSER ${ }^{1}$, MOHAMMAD SAFIUDDIN ${ }^{2}$, SHAHNAZ BINTE SAFI ${ }^{3}$ \\ ${ }^{1}$ Department of Cardiology, National Centre for Control of Rheumatic Fever and Heart Disease, Dhaka, ${ }^{2}$ Department of Cardiology, Bangabandhu Sheikh Mujib Medical University, Dhaka, ${ }^{3}$ Bangladesh Medical College \& Hospital, Dhaka \\ Address of Correspondence: Dr.Mohammad Abu Kauser, Assistant Professor of Cardiology, National Centre for Control of Rheumatic Fever and Heart Disease, Dhaka, E-mail: abukauser12@gmail.com
}

The definition and classification of heart muscle diseases has seen a notable and evolving history. Chronic myocarditis was the only recognized cause of heart muscle disease in the 1850s. In 1900 the designation of Primary myocardial disease was first introduced. However it was not until 1957, the term cardiomyopathy was used for the first time.

The term cardiomyopathy constitutes a group of disease in which the dominant feature is involvement of heart muscle itself.

For more than 30 years the term cardiomyopathy has been used to describe disorders of the heart with particular morphological and physiological characteristics. ${ }^{3,6,7}$ A variety of schemes has been proposed for classifying the cardiomyopathies, such as-

1. Functional classification

2. Etiologic classification

3. Endomyocardial biopsy/ histology

4. International classification of diseases (ICD-9/10)

5. Therapeutic classification

6. Gene based classification

Since the first WHO official classification ${ }^{1} 1$ tremendous progress has been made. ${ }^{2}$

Novel entities have been discovered requiring an update of the WHO classification in $1995^{3}$, the etiology of many forms has been clarified .

Among the above classifications no single classification of cardiomyopathies is generally accepted within the biomedical community.

In 2006,the AHA task published a new definition and classification of cardiomyopathy-called AHA 2006 scientific statement. ${ }^{4}$

The working geoup of myocardial and pericardial disease of the Europian Society of Cardiology recently published a statement - Position statement different from the 2006,AHA scientific statement. ${ }^{5}$

Functional classification

Functional classification( Table- 1) - recognizes 3 functional disturbances-

- Hypertrophy

- Dilatation

- Restriction.

Dilatation -is dominated by LV cavity enlargement and systolic failure

Hypertrophy- is dominated by LV wall thickening and diastolic failure.

Restriction-inadequate compliance causing restriction of diastolic filling.

The value of this functional classification is that virtually all cardiomyopathies are readily placed or categorized in one of the three categories and the therapeutic approaches to each category are dominantly different.

This Functional classification has some short comings also-

1. Many diseases are physiologically are heterogeneous

2. Almost all HCM have an element of diastolic restriction

3. Most DCM display myocytic hypertrophy

4. Some diseases change from one category to another during their course.

Despite this short comings Functional classification remains the most popular among the clinicians because it is based on easily understood physiologically and is relevant to therapy. 
Table-I

Functional classification

1. Cardiac dilatation

A. With systolic failure

a. Idiopathic dilated cardiomyopathy

b. Late cardiac amylodosis failure

B. Without systolic failure

a. High cadiac output state

b. Bradycardia induced congestive

heart failure

II. Cardiac hypertrophy

A. With obstruction

a. Hypertrophic obstructive cardiomyopathy

B. With out obstruction

a. Hypertrophic cardiomyopathy

b. Left ventricular Hypertrophy due

to systolic hypertension

III. Cardiac restriction

A. Early cardiac amylodosis

B. Endomyocardial fibrosis

\section{Etiologic classification}

Etiologic classification( Table- 2) - utilizes the known causes of cardiomyopathies.It has got some disadvantages also-

1. Many cases the etiologic agent is poorly understood

2. It is too long classification

3. Most similarly classified disorders are anatomically, physiologically and Therapeutically unrelated. So routinely not used.

\section{Table-II}

Etiologic classification

I. Infective/ Inflamatory

II. Metabolic

A. Endocrine

1. Thyroid diseases-

a. Thyrotoxicosis b.Hypothyroidism

2. Pheochromocytoma

3. Acromegaly.

4. Diabetes mellitus

5. Carcinoid heart disease

B. Uremia

C. Hyperoxaluria

D. Gout
E. Storage diseases \& Infiltrative processes

F. Deficiencies

1. Electrolytes

2. Nutritional

III. Amyloid

IV. General system disorder
A. Collagen vascular
B. Sarcoidosis
C. Neoplastic

V. Muscular dystrophies,Myopathies and Neuromuscular disorders
A. Muscular dystrophies
B. Congenital Myopathies
C. Mitochodrial Myopathies
D. Neuro- muscular disorders

VI. Toxicity,Hypersensitivity and Physical agent effects

A. Toxicity

B. Hypersensitivity effects

C. Physical agent effects

VII. Miscelleneous

Peripartum heart disease

Tachycardia induced cardiomyopathy etc

In Endomyocardial biopsy/histology classification In Endomyocardial biopsy/histology classification

(Table- 3): Anti-mortem histology diagnosis is used. But it has got limitations also-

1. Histology in most patients of cardoimyopathy is non specific and non diagnostic

2. Hypertrophy or fiber attenuation and fibrosis may be seen in varying degrees in all most any disorders.

3. Completely normal histology may occasionally be seen in biopsy of some cases histology

4. Myocardial biopsy sample can be subjected to several additional analytic techniques that expand the potential for classification.

Table-III

In Endomyocardial biopsy/histology classification

I. Inflamatory/Immune cardiomyopathy

II. Infectious. cardiomyopathy

A. Collagen vascular

B. Sarcoidosis

C. Neoplastic

III. Infiltrative cardiomyopathy

Glycogen storage 
Hemochromatosis

Right ventricular lipomatosis

Amylodosis

IV. Cardiac tumors - cadiag non cardiag origin

V. Miscelleneous specific cardiomyopathy

Anthacygline cardiotoxigity

Endocardial fibrosis

Endocardial fibroelastosis

Cardinoid disease

Chloroquine cardiomyopathy

Hypereonophilic syndrome

Irradiation injury

Henoch Schonlein purpyra

VI. Nonspecific abnormalities

Idiopathi dilated cardiomyopathy

Other cardiomyopathies of unknown cause

VII. No histologic abnormality

International classification of diseases

(ICD-9/10)-

International classification of diseases (ICD-9/10)-

This International classification of diseases system (Table- 4 \& Table- 5): was developed by WHO in 1948 for registering disease incidences.

US National Centre Of Health Statistics modified the ICD-9 code to allow coding of medical records.

But it is impossible to remember the long listed code numbers, so difficult to use by the physician and also the scientist.

\section{Table-IV}

International classification of diseases (ICD-9)

ICD-9 code Description

402.00 Hypertensive Heart disease, malignant w/ o $\mathrm{CHF}$

402.01 Hypertensive Heart disease, malignant w $\mathrm{CHF}$

402.10 Hypertensive Heart disease, benign w/o $\mathrm{CHF}$

402.11 Hypertensive Heart disease, benign w CHF

$422.90 \quad$ Acute myocardities

$422.91 \quad$ Idiopathic myocardities

$425.00 \quad$ Endmyocardial fibrosis

425.01 Hypertrpphic Obstructive Cardiomyopathy

$425.02 \quad$ Obscure Cardiomyopathy

$425.04 \quad$ Idopathic Cardiomyopathy

425.05 Alcoholic Cardiomyopathy
425.07 Nutritional and metabolic Cardiomyopathy

425.09 Secondary Cardiomyopathy

$674.84 \quad$ Post partumCardiomyopathy

II. Metabolic

A. Endocrine

1. Thyroid diseases-

a. Thyrotoxicosis b.Hypothyroidism

2. Pheochromocytoma

3. Acromegaly.

4. Diabetes mellitus

5. Carcinoid heart disease

B. Uremia

C. Hyperoxaluria

D. Gout

E. Storage diseases \& Infiltrative processes

Table-V

International classification of diseases (ICD-10)

ICD-9 Description

code

125.5 Ischaemic Cardiomyopathy

142 Cardiomyopathy

142.0 Dilated Cardiomyopathy

142.1 Hypertrpphic Obstructive Cardiomyopathy

142.2 Other Hypertrpphic Cardiomyopathy

142.5 Other restrictive Cardiomyopathy

142.6 Alcoholic Cardiomyopathy

142.7 Cardiomyopathy due to drugs and other external agents

142.8 Other Cardiomyopathies

142.9 Cardiomyopathy, unspecified

090.3 Cardiomyopathy in the puerperium

\section{Therapeutic classification}

Therapeutic classification( Table- 6): based on specific therapies borrows heavily from the functional and the etiologic classification of cardiomyopathy.

Nevertheless this classification also some shortcomings and so difficult to use.

1. More than one class of therapy is appropriate for a disease.

2. Therapeutic preferences are subject to variances in opinion and to change with new approaches.

3. When new therapy introduced, the existing classification become obsolete.

4. Commonly used therapies such as-inotropic agents and cardiac transplantation therapy are not included in this classification. 
Table-VI

Therapeutic classification

I. Reduced ventricular afterload

Idopathic dilated Cardiomyopathy

Late cardiac amylodosis

II. Reduced ventricular Preload

Endocardial fibrosis

Early cardiac amylodosis

III. Increase ventricular compliance

Hypertrpphic Cardiomyopathy

IV. Relieve ventricular obstruction

Hypertrpphic Obstructive

Cardiomyopathy

V. Improve cardiag rhyrthm

Cardiomyopathy of persistent tachycardia

Systemic lupus erythromatosus

VI. Speciffic therap
A. Replace deficiency
Carnitine deficiency Cardiomyopathy
B. Remove toxic agent
Hemochromatosis
Hypersensitivity
C. Immunosupression
Giant cell myocarditis
Lymphocytic myocarditis
D. Correct systemic disease
Uremic cardiomyopathy
Cardiomyopathy of cancer
E. Modulate neurohomonal environment
Idiopathic Dilated Cardiomyopathy
Most causes of congestive heart failure

\section{Former WHO classification'1980}

In spite of some objections, the most widely recognized classification of cardiomyopathies was Former WHO classification of cardiomyopathies of 1980.(Table:)

In this Former WHO classification( Table- 7): -

- Cardiomyopathies were defined as Primary myocardial disorder of unknown cause i.e. the term cardiomyopathy was reserved for the myocardial disease of unknown cause.

- Specific heart muscle disease- Heart muscle disorders of known cause called Specific heart muscle disease

- Myocardial diseases due to valvular disease, systemic hypertension, coronary artery disease and pericardial disease were excluded from this classification.
Table-VII

Former WHO classification

A. Heart muscle disease of unknown cause

Dilated Cardiomyopathy

Hypertrpphic Cardiomyopathy

Restrictive Cardiomyopathy

Unclassified Cardiomyopathy

B. Specific heart muscle disease

I. Infective

2. Metabolic

a. Endocrine

b. Familiaql storage diseases and Infiltrations

c. Deficiency

d. Amyloid

3. General system disease

a. Connective tissue disorders

b. Infiltrations and granulomaations

4. Heredofamilial

a. Muscular dystrophies

b. Neuromuscular disorders

5. Sensitivity and toxic reaction

New WHO classification of cardiomyopathies'1995

With the passage of time the distinction between primary and Specific heart muscle disease has become increasingly tenuous as the etiology and the pathogenesis of the previous idiopathic disorders has been discovered.

In 1995, WHO Committee reclassifies the cardiomyopathies- which is called New WHO classification of cardiomyopathies'1995(Table:8 )

\section{In this New WHO classification'1995-}

- Functional classification of cardiomyopathiesMyocardial diseases associated with cardiac dysfunction.

Dysfunction means abnormalities both mechanical and electrical functions.

Primary myocardial disorder of unknown cause i.e. the term cardiomyopathy of former WHO classification was changed into myocardial diseases associated with cardiac dysfunction.

-In addition to previous HCM, DCM and RCM Arrhythmogenic Right Ventricular Cardiomyopathy (ARVC)/ Arrhythmogenic Right Ventricular dysplasia (ARVD) was in the Functional class.

-Functional classification utilized two separate methods to define the individual categories. The first was based 
on the global anatomic description of chamber dimension in systole and diastole. Thus the dilated and restricted categories have definitions based on left ventricular dimension or volume.

- The second method of creating individual categories are genetically based; have unique myocardial phenotypic features and do not exhibit extra cardiac phentypes.HCM caused by mutations in contractile proteins manifesting unique phenotype. The same true for Arrhythmogenic Right Ventricular Cardiomyopathy (ARVC)

- Unclassified cardiomyopathies remains same as it was in former classification but a new variety of cadiomyopathy named 'Left ventricular Non Compacted myocardium (LVNC)/ Non compaction of left ventricular myocardium' was included in the Unclassified cardiomyopathies..

-Specific cardiomyopathies -Specific heart muscle diseases of former WHO classification was changed into Specific cardiomyopathies.

The concept of Specific cardiomyopathies was widened too much so as to include myocardial diseases due to valvular disease, systemic hypertension; coronary artery disease and pericardial disease were also included as Specific cardiomyopathies.

-Myocarditis also included in these Specific cardiomyopathies as inflammatory cardiomyopathy.

Although the 1995 classification made significant contribution (acknowledging new entities, unifying terminology including myocarditis) nonetheless it introduced ambiguities by classifying the ischemic and overload disorders among the cadiomyopathies.

Table-VIII

New WHO classification

A. Functional classification of cardiomyopathy

Dilated Cardiomyopathy

Hypertrpphic Cardiomyopathy

Restrictive Cardiomyopathy

Unclassified Cardiomyopathy

B. Specific Cardiomyopathy

1. Ischaemic Cardiomyopathy

2. Valvular Cardiomyopathy

3. Hypertansive Cardiomyopathy

4. Inflamatory Cardiomyopathy
a. Idiopathic
b. Autoimmune
c. Infectious

5. Metabolic Cardiomyopathy

a.Endocrine

b. Familial storage diseases and Infiltrations

c. Deficiency

d. Amyloid

6. General system disease

a. Connective tissue disease

b. Infiltrations and Granulations

7. Muscular dystrophies

8. Neuromuscular disorders

9. Sensitivity and toxic reaction

10. Peripheral neuropathy

\section{AHA scientific statement,}

Each of the previous proposed definitions and classifications through years have some shortcomings.

In particular the popular hypertrophic -dilated restrictive cardiomyopathies classification has major limitations by virtue of mixing anatomic designations (i.e.hypertrophic and dilated) with functional one (i.e. restrictive). Consequently confusion arises when the same disease could appear in or even three categories and some diseases do not have uniformly static expression and may evolve from one category to another during their clinical course. For example HCM, Amyloid and other infiltrative myocardial conditions may progress from a non dilated state with ventricular stiffness to a dilated form with systolic dysfunction and heart failure.

Etiologic classifications of cardimyopathies are also problematic given that the diseases with the same / similar phenotypes can harbor diverse etiologies and mechanisms. For example-DCM may genetic, infective, autoimmune and toxic causes (and in some cases are still designated as idiopathic) all leading, however to the final common pathway of ventricular dilatation with systolic dysfunction.

However with the identification of new diseases over the past decades and recent advances in cardiovascular diagnosis and knowledge regarding etiology, some diseases definitions have become outdated and past classification rendered essentially obsolete. 
The great advances in the field of cardiomyopathies came from the rapid revolution of molecular biology in cardiology made fundamental step forward in understanding the genetics of cardiomyopathies. In particular ion channelopathies have emerge $d$ as condition predisposing to potentially lethal ventricular tachyarrhythmia and by mutations in proteins leading to dysfunctional Sodium, Potassium, calcium and other ion channels.

In 2006, the AHA Task force incorporated the above ideas in a scientific statement - called AHA 2006 scientific statement, advancing a new definition and classifications.

The AHA task force proposed a 'beyond of phenotype' classification of cardiomyopathies that groups under such a designation all myocardial diseases whose common denominator is a genetic defect, consisting of mutants gene encoding sarcomeric, cytoskeleton ,desmosomal or ion channel proteins.

Accordingly the AHA task force proposed following definition of cardiomyopathy-

Cardiomyopathies are a heterogeneous group of diseases of the myocardium associated with mechanical and/or electrical dysfunction that usually (but not invariably) exhibit inappropriate ventricular hypertrophy and dilatation and are due to a variety of causes that frequently are genetic.

In AHA scientific statement, the original pmimary and secondary forms of cardiomyopathy was reintroduced but with slight modification of definition .

Primary cardiomyopathies are those solely or predominantly confined to the heart muscle .

Primary cardiomyopathies have been divided into three main categories (Table: 9)

- Genetic

- Mixed (genetic and nongenetic)-

- Acquired

In genetic-In addition to HCM,ARVC/ARVD, LVNC the followings are also included-Primary electrical disorders with structural normal heart i.e. Ion channel disorders (LQTS,SQTS ,Brugada syndrome,CVPT ,Asian SUNDS

In Mixed (genetic and nongenetic)-DCM,-RCM

(Non-hypertropied \& non dilated)
In Acquired- Myocarditis was added as inflammatory cardiomyopathy. Besides in acquired cardiomyopaythy, Peripartum Cardiomyopaythy, Tachycardia induced cardiomyopathy, Tako-tsubo or stress provoked cardiomyopathy also added.

Secondary cardiomyopathies are those involve the heart muscle as a part of generalized systemic (multi-organ) disorders .

N.B: Unclassified variety of cardiomyopathy of previous WHO classification, discarded from these AHA classification.

Secondary cardiomyopathies- included the common disorders that frequently associated with cardiomyopathies. Lists of disorders are shown in the table ( Table-9.1 )

Table-IX

Primary cardiomyopathies of AHA scientific statement

\begin{tabular}{c}
\hline Genetic - HCM \\
ARVC/D \\
LVNC \\
Glycogen storage \\
PRKAG2 \\
Danon \\
Conduction defect \\
MitochondriaLmyopathies \\
Ion channel disorders \\
$\quad$ LQTS \\
$\quad$ Brugada \\
SQTS \\
CVPT \\
Asian SUNDS \\
Mixed (genetic and monogenetic) \\
DCM \\
RESTRICTIVE \\
(Nonhypertrophied and non-dilated) \\
INFLAMATORY \\
STRESS PROVKED-Tako-tsubo \\
PERIPARTUM \\
TACHYCARDIA INDUCED \\
Acquired of Insulin Dependent Diabetic Mother \\
\hline
\end{tabular}

HCM=Hypertophic CardioMyopathy. ARVC/D= arrhythmogenic right ventricular cardiomyopathy/ dysplasia ,LVNC =left ventrcular noncompactin

LQTS = long QT syndrome, Brugada syndrome

SQTS= short QT syndrome,CVPT=catecholaminergic polymorphic ventricular tachycardia,Asian SUND =Asian unexplained natural death syndrome 
Table-IX (i)

Secondary cardiomyopathies of AHA scientific statement

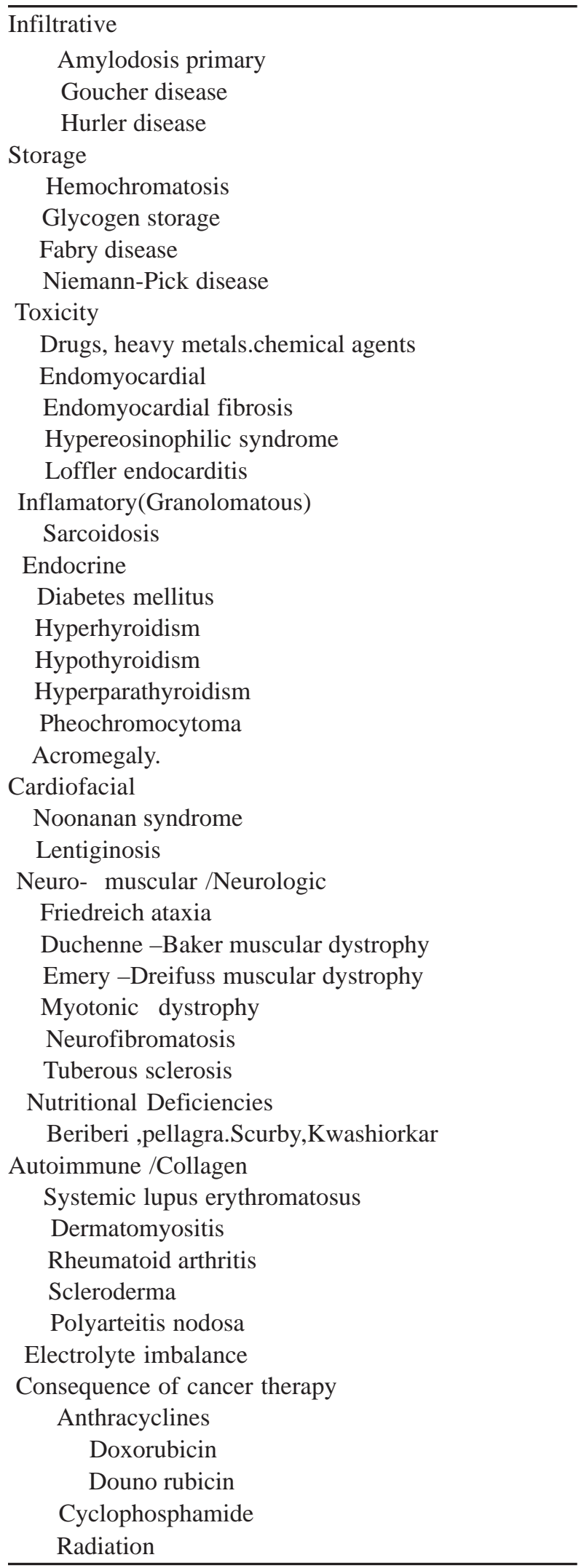

\section{ESC position statement}

The rationale for a new classification: With the advancement of molecular biology, Expert committee of AHA proposed new definition and classification of cardiomyopathy.Primary and secondary cardimyopathies as describes in AHA proposed new definition and classification, the challenges of distinguishing primary and secondary cardiomyopathies is still remains controversial because- many of the diseases classed as proposed primary cardiomyopathies can be associated with major extra-cardiac manifestations and conversely pathology in many of the diseases classed as secondary cardiomyopathies can predominantly (or exclusively) involve the heart.

As many cardiomyopathies are caused by mutations in genes that encode various cardiac proteins, an alternative approach is to classify cardiomyopathies according to the causative genetic defect.

However in clinical practice the pathway from diagnosis to treatment rarely begins with the identification of an underlying genetic mutation; more usually patients present with symptoms or are incidentally found to have clinical signs or abnormal screening tests. Even when the genetic defect is known in a family, the identification of clinically relevant disease in gene carriers still requires the demonstration of a morphological phenotype.

Thus the clinically oriented classification system in which heart muscle disorders are grouped according to ventricular morphology and function remains the most useful method for diagnosing and managing patients and families with heart muscle disease.

Considering the above mentioned situations, In 2007 ESC working group on myocardial and pericardial diseases published a new classification of cardiomyopathies, known as 2007 ESC. position statement. .(Table no: 10 )

\section{In this statement cardiomyopathy is defined as-}

A myocardial disorder in which the heart muscle is structurally and functionally abnormal in the absence of coronary artery disease, hypertension, valvular heart disease and congenital heart disease sufficient to cause the observed myocardial abnormality.

In this statement Cardiomyopathies are grouped into 5 specific morphological and functional phenotypes.

Each phenotype is again sub classified into two formsFamilial and Non-familial. Each Familial is again subdivided into two forms- Familial due to unknown gene and Familial due to known gene. 
Familial-clinically defined by the occurrence in more than one family member of either the same disorder or phenotype. That is (or could be) caused by the same genetic mutation and not to acquired cardiac or systemic diseases in which the clinical phenotype is influenced by genetic polymorphism.

While accepting and reinforcing the idea advanced by AHA statement to divide cardiomyopathies into familial/ genetic and non-familial / nongenetic the traditional division of primary and secondary cardiomyopathies have abolished in the position statement.

Most familial cardiomyopathies are monogenic disorder i.e. The gene defect is sufficient by itself to cause the trait. Familial category can be subsequently transmitted to their offspring.

Non-familial-clinically defined by the presence of a cardiomyopathy in the index patient and the absence of disease in the other family members.

Each Non familial is again subdivided into two formsNon familial due to unknown gene and Non familial due to known gene.

Moreover ,the concept of pure electrical dysfunction was denied ,thus ruling out ion channel and conduction system disease from this classification, because recent studies suggest that genes encoding ion channels may be implicated in subgroups of patients with DCM ,conduction disorders and arrhythmias do not provide an argument for the redesignation of channelopathies as cardiomyopathies at the present time.

\section{Table-X}

Proposed new classification of CardiomyopathiesEuropian society of Cardiology.

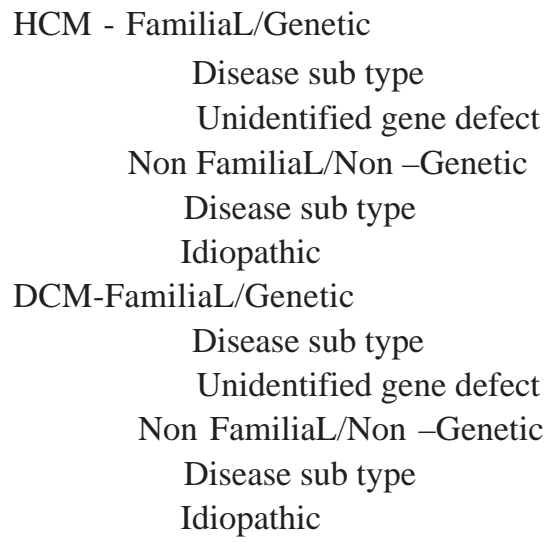

table continued

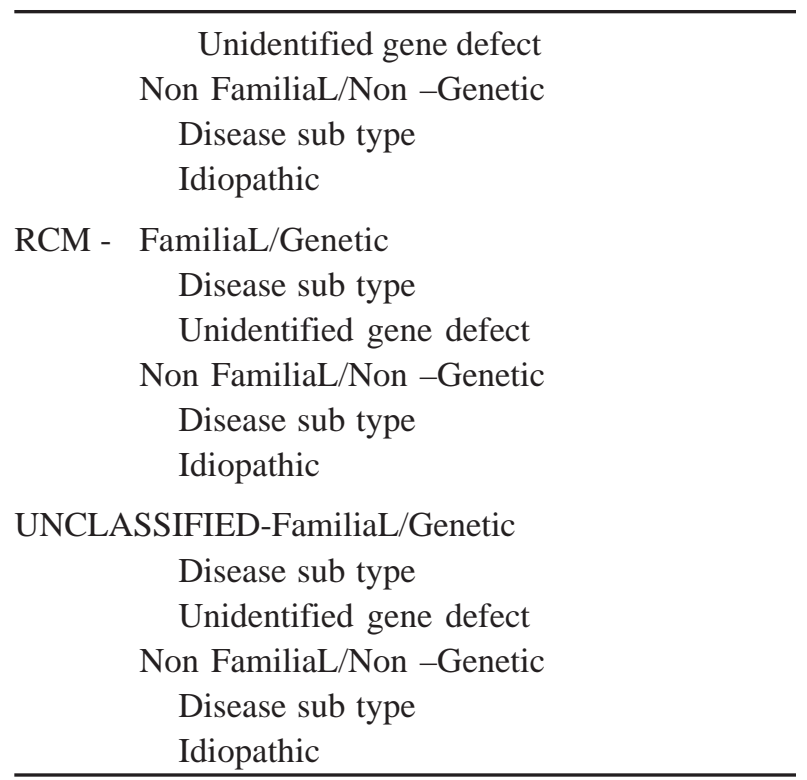

What is new in 2007 ESC. position statement.

The proposed 2007 ESC Position statement is designed to provide a valid tool for clinical practice.

This proposal differs in several ways from the previous several classifications including 2006 AHA scientific statement-

\section{This classification-}

- Based on specific morphological and functional phenotypes rather than putative pathophysiological mechanism.

- Classified into familial and non familial forms so as to raise the awareness of genetic determinants of cardiomyopathies and to orient diagnostic test (including the search for specific mutations when appropriate)

- Again discard the term primary and secondary form of cardiomtopathies.

- Reintroduction of the unclassified variety of cardiomyopathy.

\section{Limitations of 2007 ESC. position statement.}

1. There are circumstances in which this classification system fails to describe fully the complexity the of some disease phenotypes such as-

- Occurrence of different cardiomyopathies caused by the same genetic mutations (in related and unrelated individuals).

- The evolution one disease phenotype into another overtime. 
2. Another limitation of the proposed classification is the problem of the mixed phenotype,for example -in patient with dilated and hypertrophied ventricles

3. This proposed classification does not provide guidance on the diagnostic algorhythm that should be followed whenever a cardiomyopathy is diagnosed.

\section{Conclusion:}

Indeed no past or present classification of cardiomyopathies is likely to satisfy the purposes of all interested parties. The aim of this classification is to help clinicians look beyond generic diagnostic labels in order to reach more specific diagnosis that may be useful for tailored clinical management of patients and their families.

2007 ESC position statement working group hope that subsequent revisions of the classification based on emerging data will resolve remaining shortcomings and ambiguities.

\section{References}

1. Report of the WHO/ISFC Task force on the definition and classification of cardiomyopathies.Br Heart J 1980;44:672-673.

2. Thiene G.Basso C,Calabrese F,Angelini A, Valente M. Twenty years of progress and beckoning frontiers in cardiovascular pathology: cardiomyopathies. Cardiovasgular patho 2005;14:165-16.

3. Richardson P,McKenna W,Britow M,Maisch B, Mautner B, O’Connel J, Olsen E, Thiene G, Goodwin J,Gyarfas I.Martin I,Nordet P.Report of the 1995 WHO/ISFC Task force on the definition and classification of dardiomyopathies..Circulation 1996;93:841-842.

4. MaronBJ,TowbinLA,Thiene G, Antzelevitch C,Corrado D, Arnett D,Moss AJ,Seidman CE, Young JB,American Heart Association.Council on clinical cardiology,Heart failure and Transplantation committee.......American Heart Association Scientific Statement.Circulation 2006;113:1807-1816.

5. Elliott P,Andersson B, Arbustini E, Bilinska Z,Cecchi F,Charron P,Dubourg O,Khul U, Maisch B, McKenna WJ, Monserrat L,Oankuwrit S, Rapezzi C,Sefeovic P,Tavazzi LKeren A. Classification of cardiomyopathies: a position statement from the Europian Society of CardiologyWorking Group on Myocardial and Pericardial diseases. Eu Heart J;2008:29:270-27.

6. Goodwin JF .The frontiers of cardiomyopathy..Br Heart J;1982:48:1-18

7. Abelmann WHO.Classification and natural history of primary myocardial disease.Prog Cardiovasc Dis 1984;27:73-94 